\title{
(More) About biphenyl first excited triplet state energy
}

\author{
Paulo J.S. Gomes, Carlos Serpa*, Luis G. Arnaut \\ Department of Chemistry, University of Coimbra, 3000-535 Coimbra, Portugal \\ Received 14 March 2006; accepted 20 April 2006 \\ Available online 28 April 2006
}

\begin{abstract}
Biphenyl photophysics was extensively studied in the past and much attention was given to the measurement of its first excited triplet state energy. Phosphorescence and the corresponding $\mathrm{T}_{1} \leftarrow \mathrm{S}_{0}$ absorption give triplet energies that differ by $10 \mathrm{kcal} \mathrm{mol}^{-1}$. We revisited biphenyl photophysics using photoacoustic calorimetry (PAC), a technique that directly measures the thermochemistry and kinetics of short-lived transients in solution. PAC measurements give the relaxed biphenyl triplet energy of $67.8 \pm 0.6 \mathrm{kcal} \mathrm{mol}^{-1} ; 2.3 \mathrm{kcal} \mathrm{mol}^{-1}$ higher than the spectroscopic value. The direct measurement of the biphenyl relaxed triplet energy reported in this work should resolve the continued misuse of its spectroscopic energy, in particular in the growing field of polyphenylene polymers.
\end{abstract}

(C) 2006 Elsevier B.V. All rights reserved.

Keywords: Photoacoustic calorimetry; Triplet energy; Biphenyl; Energy transfer

\section{Introduction}

Biphenyl consists of two aromatic rings connected by one covalent bond. Hindered rotation of the two phenyl rings in the ground state allows for the variation of their dihedral angle. This rotation is blocked in the triplet state because the inter-ring bond acquires some double-bond character [1], and triplet state geometry is much more rigid. Biphenyl photophysics was extensively studied in the past, both experimentally and theoretically [2-5]. In particular, much attention was given to the measurement of its first excited triplet state energy. In 1967, from concerted absorption, phosphorescence and quenching data, Wagner estimated that the true $\mathrm{T}_{1} \leftarrow \mathrm{S}_{0}$ transition of biphenyl occurs around $69.5 \mathrm{kcal} \mathrm{mol}^{-1}$ [6]. This energy neither corresponds to the highest energy phosphorescence band at $437 \mathrm{~nm}\left(65.4 \mathrm{kcal} \mathrm{mol}^{-1}\right)$ nor to the lowest energy band in the $\mathrm{T}_{1} \leftarrow \mathrm{S}_{0}$ absorption spectra $\left(376 \mathrm{~nm}, 76.0 \mathrm{kcal} \mathrm{mol}^{-1}\right)$ [7], and was assigned to a nonspectroscopic transition. Further work by Wagner on the quenching of benzophenone and other compounds by biphenyl yielded the triplet energy of $68.5 \mathrm{kcal} \mathrm{mol}^{-1}$ [8]. Gessner and Scaiano also studied the kinetics of the reaction of benzophenone and biphenyl over a temperature range, and report values of

\footnotetext{
* Corresponding author. Tel.: +351 239852080; fax: +351 239827703.

E-mail addresses: pgomes@qui.uc.pt (P.J.S. Gomes), serpasoa@ci.uc.pt (C. Serpa), lgarnaut@ci.uc.pt (L.G. Arnaut).
}

$\Delta H^{\circ}=-2 \mathrm{kcal} \mathrm{mol}^{-1}$ and $\Delta S^{\circ}=-1.8 \mathrm{cal} \mathrm{K}^{-1} \mathrm{~mol}^{-1}$ [9]. They argued for a decrease in conformational freedom upon excitation of biphenyl. Kira and Thomas measured the equilibrium constant between the biphenyl triplet and triplets of known energy, and obtained $\Delta G^{\circ}=-1.87 \mathrm{kcal} \mathrm{mol}^{-1}$ for benzophenone/biphenyl equilibrium [10]. Assuming that the energy difference between the triplets is equal to $\Delta G^{\circ}$, they reported the triplet energy of $67.6 \mathrm{kcal} \mathrm{mol}^{-1}$ for biphenyl. A different approach, using a photothermal method, was followed by Poston and Harris [11] that studied the decay of the lowest excited triplet state of benzophenone and its energy transfer to biphenyl. They reported an energy of $67 \pm 0.5 \mathrm{kcal} \mathrm{mol}^{-1}$ for the biphenyl triplet state and an energy transfer rate from benzophenone to biphenyl of $(4.7 \pm 0.8) \times 10^{8} \mathrm{M}^{-1} \mathrm{~s}^{-1}$ in carbon tetrachloride. They argued for the establishment of an equilibrium between this "direct" energy transfer and the reverse process. The measured enthalpy was identical to that obtained by Gessner and Scaiano.

Energy transfer studies involving biphenyl often rely on its spectroscopic triplet energy, and the triplet energies of other molecules obtained by this method are biased by the use of the spectroscopic triplet energy of biphenyl [12]. Biphenyl has a renewed significance today, since it is the repeating unit of polyphenyl films with potential application as conducting polymers $[13,14]$ and as light emitting diodes $[15,16]$. The triplet energy of biphenyl tabulated in reference databases $[17,18]$ is still its spectroscopic energy $\left(E_{\mathrm{T}}(\right.$ biphenyl $\left.)=65.5 \mathrm{kcal} \mathrm{mol}^{-1}\right)$. 
These reasons motivated our reassessment of the biphenyl triplet energy using photoacoustic calorimetry (PAC).

PAC has proven to be a very useful technique to measure the thermochemistry and kinetics of short lived transients in solution $[19,20]$. This technique detects the heat released as the excited state reached by photoexcitation decays to lower energy states, including the triplet state. We apply this technique to measure the triplet state energy of biphenyl formed by sensitization with xanthone and benzophenone. We also studied fluorene, a molecule where the presence of a methylene bridge between the two aromatic rings prevents their rotation, but that otherwise resembles biphenyl. The kinetics of energy transfer from xanthone and benzophenone to biphenyl and fluorene were further explored using laser flash photolysis. The value of $67.8 \mathrm{kcal} \mathrm{mol}^{-1}$ was obtained for biphenyl triplet energy, confirming the decrease in conformational freedom upon excitation of this molecule [21]. As expected, the spectroscopic triplet energy of fluorene and its $\mathrm{T}_{1} \leftarrow \mathrm{S}_{0}$ transition energy determined by PAC gave identical values, $66.8 \pm 0.5 \mathrm{kcal} \mathrm{mol}^{-1}$.

\section{Experimental}

Biphenyl from Aldrich was purified by vacuum sublimation. Benzophenone from Aldrich was zone refined. Best available fluorene and 2-hydroxybenzophenone from Aldrich and xanthone from Lancaster were used without any further purification. Benzene (Aldrich, HPLC grade, 99.9\%) was used as solvent. Further purification of benzene by drying and distillation over sodium did not change the solvent spectroscopic characteristics. Spectroscopic grade $n$-heptane from Aldrich was dried over sodium. 3-Methylpentane (Aldrich, 99+\%) was used without further purification.

Ground state absorption was detected with a Shimadzu UV-2100 spectrophotometer. Fluorescence and phosporescence spectra were obtained with a SPEX Fluorolog 3-22 spectroflurimeter. The 1934D4 module for phosphorimetry was used. Flash photolysis experiments employed an Applied Photophysics LKS.60 laser flash photolysis spectrometer, with a Hewlett-Packard Infinium Oscilloscope and a Spectra-Physics Quanta-Ray GCR-130 Nd:YAG laser as excitation source.

We use a front-face irradiation photoacoustic cell [20,22]. In short, the sample, reference and solvent solutions were flown separately with a $0.9 \mathrm{~mL} / \mathrm{min}$ rate (SSI chromatographic pump) through a $0.22 \mathrm{~mm}$ thick cell. They were irradiated at $337 \mathrm{~nm}$ with a nitrogen laser (PTI, model PL 2300) at a frequency of $2 \mathrm{~Hz}$. A small fraction of the laser beam was reflected to a photodiode, used to trigger the transient recorder (Tektronix DSA $601,1 \mathrm{Gs} / \mathrm{s})$. The photoacoustic waves, detected with a 2.25 MHz Panametrics transducer (model 5676) and captured by the transient recorder, were transferred to a PC for data analysis. In a typical PAC experience 100 waves of the sample, reference and pure solvent are recorded and averaged in the same experimental conditions. Four sets of averaged sample, reference and solvent waves are used for the data analysis at a given laser intensity, and four laser intensities are employed in each experiment. The different laser intensities are obtained by interposing neutral density filters with transmissions between 30 and
$100 \%$. All the measurements were made in benzene using 2hydroxybenzophenone as photoacoustic reference.

All samples used were saturated with $\mathrm{N}_{2}$ or Ar before the measurements.

\section{Results and discussion}

\subsection{Steady-state absorption and emission}

Fig. 1 presents the normalised absorption, fluorescence and low temperature phosphorescence spectra of biphenyl and fluorene. As expected from the similar structure of the two molecules, these spectra have some resemblances. The most important difference is in the absorption spectra. Fluorene absorption clearly shows a vibrational progression whereas biphenyl absorption, as a result of the geometrical freedom of the ground state of this molecule, does not have any vibrational structure. The Stokes shift from the absorption and fluorescence emission of fluorene is very small, confirming the small change in geometry from ground to excited state. From the observed maximum of the first emission band of both phosphorescence spectra we determine the spectroscopic triplet energy of $65.4 \mathrm{kcal} \mathrm{mol}^{-1}$ for biphenyl and of $67.4 \mathrm{kcal} \mathrm{mol}^{-1}$ for fluorene.

\subsection{Transient absorption}

The kinetics of the energy transfer reactions from benzophenone and xanthone to biphenyl and fluorene were studied in benzene. Fig. 2 shows the decay of the benzophenone triplet at 320 and $530 \mathrm{~nm}$ with the concomitant growth of a band at the wavelength characteristic of the fluorene triplet transient absorption, $370 \mathrm{~nm}$. In all the four reactions studied, we observed the formation of the triplet state of the quencher as the benzophenone or xanthone triplet decayed, confirming the nature of the triplet energy transfer reaction. Eq. (1) was used to determine the energy transfer rate $\left(k_{\mathrm{EnT}}\right)$. The decay rates in absence $\left(k_{0}\right)$ or presence $(k)$ of quencher were determined by fitting single exponentials to the experimental decays at the maximum of the triplet-triplet absorption bands of benzophenone and xanthone.

$k=k_{0}+k_{\mathrm{EnT}}[Q]$

The energy transfer rate constants obtained with Eq. (1) are presented in Table 1. The benzophenone quenching rate constants are slower than the xanthone ones, by one order of magnitude in the case of biphenyl. The xanthone triplet energy is $5.5 \mathrm{kcal} \mathrm{mol}^{-1}$ higher than that of benzophenone, and the

Table 1

Benzophenone and xanthone triplet energy $\left(E_{\mathrm{T}}\right)$, triplet decay rate $\left(k_{0}\right)$ and energy transfer rate $\left(k_{\mathrm{EnT}}\right)$ from sensitizer to biphenyl and fluorene, in benzene

\begin{tabular}{lllll}
\hline Sensitizer & $E_{\mathrm{T}}{ }^{\mathrm{a}}\left(\mathrm{kcal} \mathrm{mol}^{-1}\right)$ & $k_{0}\left(\mathrm{~s}^{-1}\right)$ & \multicolumn{2}{l}{$k_{\mathrm{EnT}}\left(\mathrm{M}^{-1} \mathrm{~s}^{-1}\right)$} \\
\cline { 3 - 5 } & & & Biphenyl & Fluorene \\
\hline Benzophenone & 68.6 & $2.12 \times 10^{5}$ & $6.6 \times 10^{8}$ & $2.5 \times 10^{9}$ \\
Xanthone & 74.1 & $1.07 \times 10^{7}$ & $6.2 \times 10^{9}$ & $8.1 \times 10^{9}$
\end{tabular}

a Reference [17] 
<smiles>c1ccc2ccccc2c1</smiles>
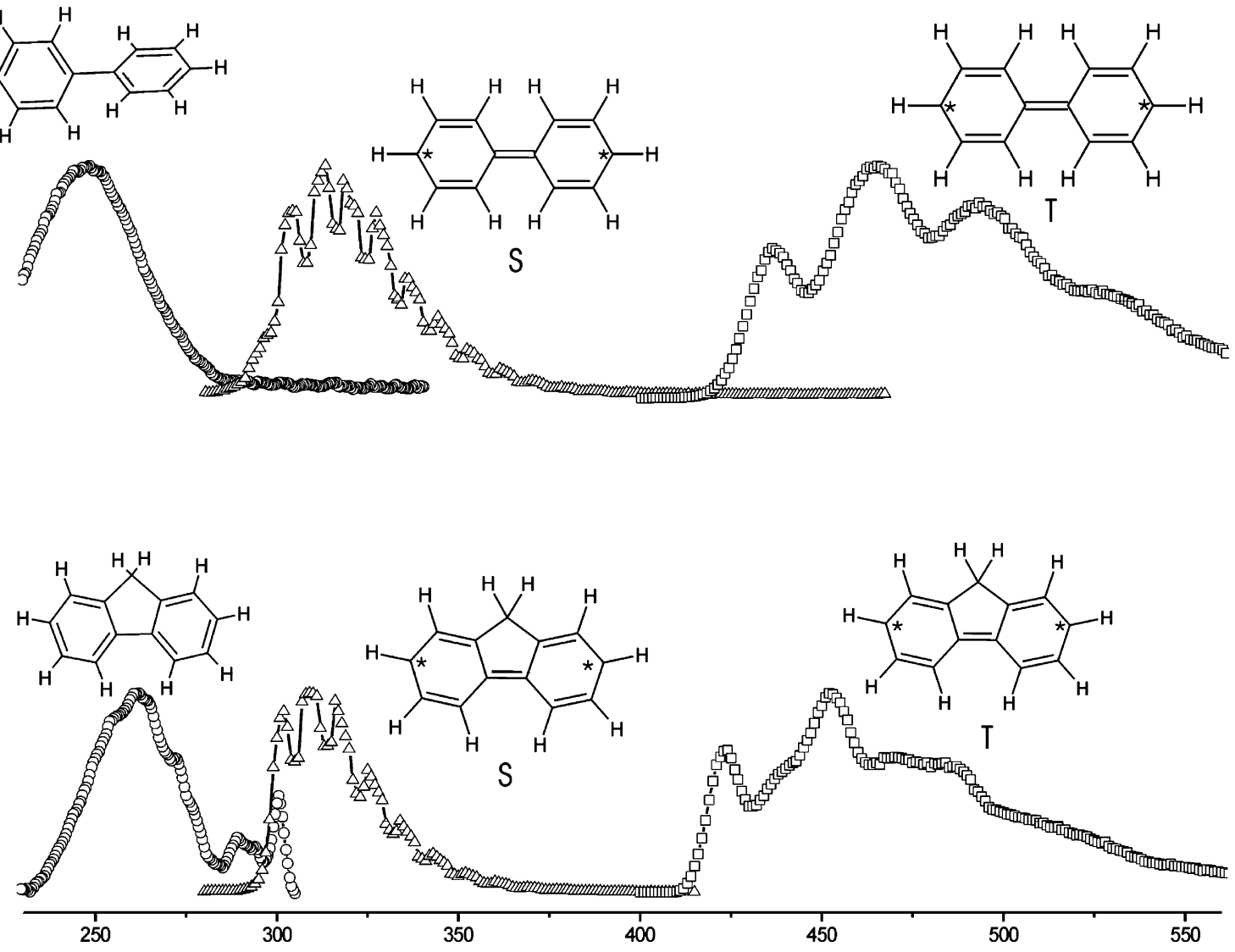

Fig. 1. Normalised biphenyl (top) and fluorene (bottom) absorption and fluorescence spectra in $n$-heptane at room temperature and phosphorescence spectrum in 3-methyl pentane at $77 \mathrm{~K}$. The corresponding structures are shown on top of each spectrum.

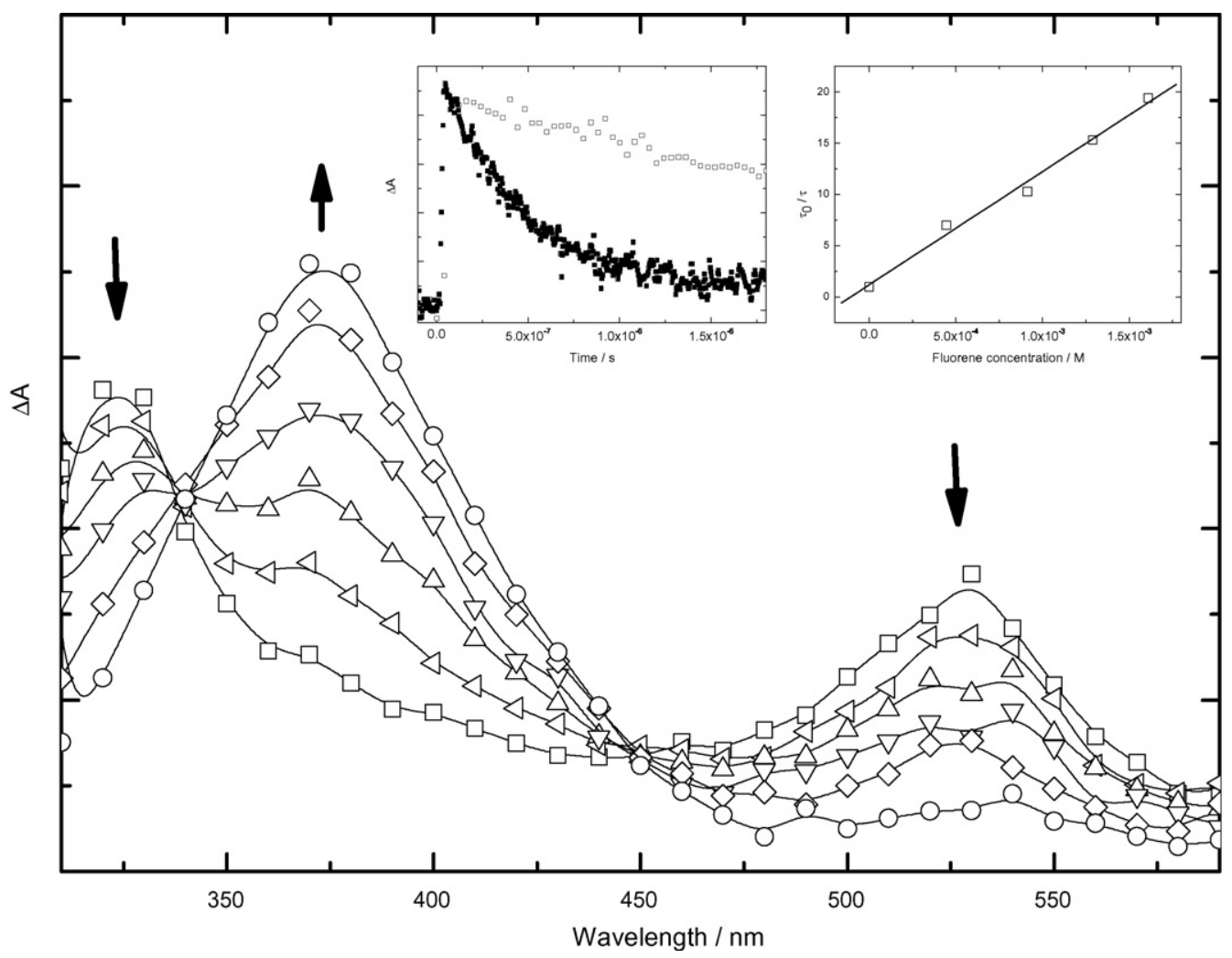

Fig. 2. Transient spectra for benzophenone in presence of fluorene ([fluorene] $=9.14 \times 10^{-4} \mathrm{M}$ ). Times from squares to circles: $120 \mathrm{~ns}, 200 \mathrm{~ns}, 280 \mathrm{~ns}, 400 \mathrm{~ns}, 600 \mathrm{~ns}$ and $1 \mu \mathrm{s}$. Inserted: kinetic decays obtained at $530 \mathrm{~nm}$ (left) and the Stern-Volmer plot for different fluorene concentrations (right). 


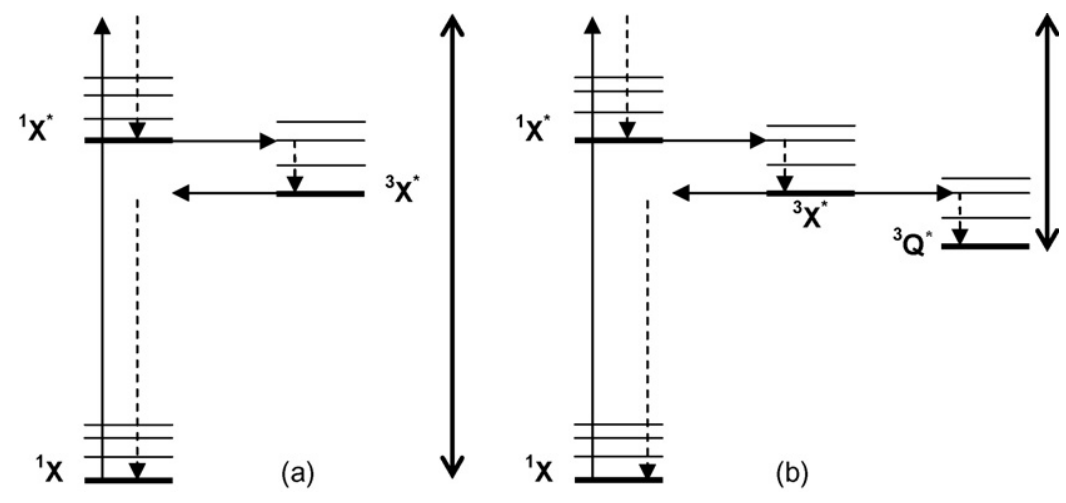

Scheme 1. Excitation and decay processes of xanthone (X) in absence (a) and presence (b) of a suitable triplet state quencher (Q).

faster rates observed with xanthone are consistent with their larger exothermicity. The rate constant for the quenching of xanthone by fluorene approaches the diffusion limit in benzene, $1.0 \times 10^{10} \mathrm{M}^{-1} \mathrm{~s}^{-1}$ [17], and may be the reason why the difference with the quenching rate of benzophenone is not as pronounced for fluorene as it is for biphenyl.

\subsection{Photoacoustic calorimetry}

Photoacoustic calorimetry has provided accurate determinations of triplet state energies of various organic molecules [19]. A rather comprehensive study of alkene triplet energies was done by Caldwell and co-workers, and permitted to assess the energy differences between relaxed and spectroscopic triplets [23-25]. We have also used this technique to study the triplet energies of organic polymers [26,27], the enthalpy of electron transfer reactions $[22,28]$, and of the photocyclization of phenylamines [29,30].

Photoacoustic calorimetry detects the heat released in the decay of an excited state. The transient heat produces an acoustic wave that is detected by a high-frequency transducer. The deconvolution of the acoustic waves requires the knowledge of the decay mechanism. Our transient absorption experiments inform on that mechanism. After excitation in benzene, the first excited singlet state of xanthone is formed via internal conversion. Then, intersystem crossing to the triplet state occurs with unit quantum yield. This triplet state is formed in less than $1 \mathrm{~ns}$ [31]. The xanthone triplet state lifetime in benzene, as measured by triplet-triplet absorption, is $94 \mathrm{~ns}$. As phosphorescence is negligible at room temperature, all the excitation energy is released non-radiatively within $100 \mathrm{~ns}$ after excitation (Scheme 1a). PAC detects this heat release and may resolve its kinetics.

If a quencher is present in solution, a new deactivation route in opened. Thus, adding biphenyl or fluorene to xanthone in solution will shorten its lifetime. The kinetics of the energy transfer reactions are known from the transient absorption spectroscopy measurements. The fraction of heat $\left(\phi_{\mathrm{PAC}}\right)$ detected in the presence of a quencher is described by Eq. (2) (Scheme 1b). We have used sufficient high concentrations of biphenyl and fluorene to quench at least $94 \%$ of the xanthone triplets. Under these conditions biphenyl or fluorene triplets are formed very fast and with almost unit efficiency, but decay very slowly, with lifetimes (more than $100 \mathrm{~ms}$ [17]), beyond our time resolution. Thus, PAC measures directly the heat released in the formation of the quencher triplet, following energy transfer from the sensitizer (Scheme 1b). Knowing the excitation energy $\left(E_{h v}\right)$ and xanthone triplet energy $\left(E_{\mathrm{T}(\mathrm{X})}\right)$, and determining the yield of energy transfer $\left(\Phi_{\text {EnT }}\right)$ by independent transient absorption experiments, the quencher relaxed triplet energy $\left(E_{\mathrm{T}(\mathrm{Q})}\right)$ is given by

$$
\begin{aligned}
\phi_{\mathrm{PAC}} E_{h v}= & \left(E_{h v}-E_{\mathrm{T}(\mathrm{X})}\right)+\left(1-\Phi_{\mathrm{EnT}}\right) E_{\mathrm{T}(\mathrm{X})} \\
& +\Phi_{\mathrm{EnT}}\left(E_{\mathrm{T}(\mathrm{X})}-E_{\mathrm{T}(\mathrm{Q})}\right)
\end{aligned}
$$

Fig. 3 shows the solvent-subtracted and normalised photoacoustic waves obtained for xanthone and for xanthone in presence of biphenyl, in benzene. All the energy absorbed by the photoacoustic reference (2-hydroxybenzophenone, HBP) is very rapidly deposited as heat in the medium (in less than $40 \mathrm{ps}$ in hexane [32]). The corresponding acoustic wave is also shown in Fig. 3. The photoacoustic waves of xanthone and HBP are clearly dephased in time. This indicates that the heat released as the xanthone triplet decays is, as expected, much slower than the reference heat deposition. The dephasing between reference

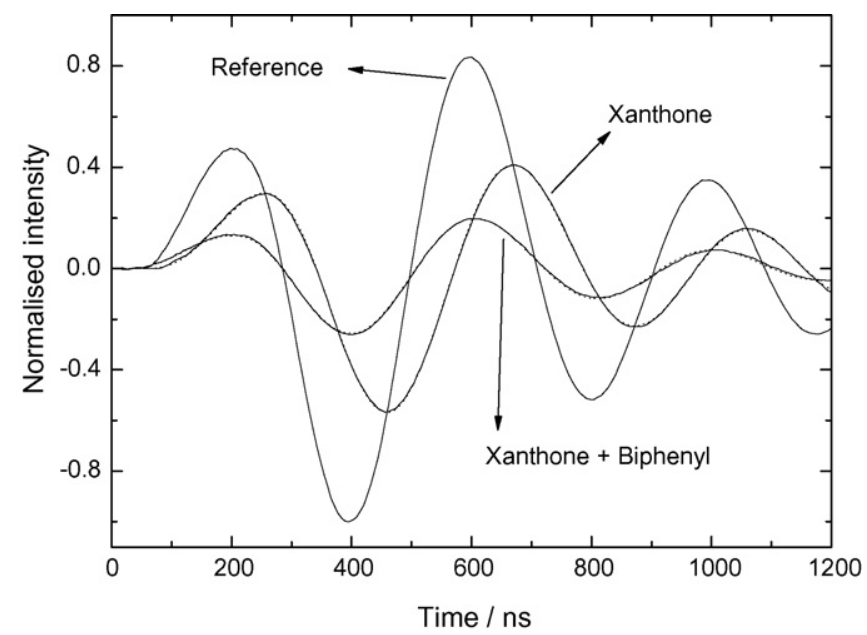

Fig. 3. Normalised PAC waves for xanthone and xanthone in presence of biphenyl, in benzene ([biphenyl] $=0.0492 \mathrm{M}$ ). Also showed the normalised wave for the reference. Dotted lines are the calculated waves (see text). 
Table 2

Photoacoustic calorimetry of xanthone + biphenyl or xanthone + fluorene, in benzene ${ }^{\mathrm{a}}$

\begin{tabular}{|c|c|c|c|c|c|c|}
\hline Concentration (M) & $\Phi_{\mathrm{EnT}}$ & $1-\Phi_{\mathrm{EnT}}$ & $\tau(\mathrm{ns})$ & $\tau_{\mathrm{PAC}}(\mathrm{ns})$ & $\phi_{\mathrm{PAC}}$ & $E_{\mathrm{T}}\left(\mathrm{kcal} \mathrm{mol}^{-1}\right)$ \\
\hline 0 & 0 & 1 & $93.5^{\mathrm{b}}$ & 94.4 & 1.06 & - \\
\hline 0.0293 & 0.944 & 0.056 & 5.2 & 1.2 & 0.236 & 68.4 \\
\hline 0.0700 & 0.976 & 0.024 & 2.3 & 1.0 & 0.226 & 67.3 \\
\hline \multicolumn{7}{|l|}{ [Fluorene] } \\
\hline 0.0789 & 0.984 & 0.016 & 1.5 & 1.3 & 0.230 & 66.4 \\
\hline
\end{tabular}

a The values of $\tau$ and $\Phi_{\text {EnT }}$ were obtained using Eq. (1).

b Experimental value obtained from the transient absorption for xanthone.

and sample waves almost disappears in the presence of a high concentration of biphenyl, and the amplitude of the sample wave is reduced by a factor of 4 . This is the behaviour expected from reduction of the xanthone lifetime to $3 \mathrm{~ns}$ and from the $97 \%$ energy transfer from the xanthone triplet to the biphenyl triplet that does not decay within the time resolution of our experiment.

The reference and sample photoacoustic waves were deconvoluted using an implementation of the Marquardt algorithm [20], using the kinetic model described above. All waves were interpreted in terms of two sequential decays, each one described by the fraction of thermal heat deposited, $\phi$, within the lifetime $\tau$. We did not use any restrictions in the fitting of the four parameters used. Fig. 3 includes the waves calculated with the two fractions of heat released in the corresponding lifetimes, obtained in the deconvolution process. The appropriateness of our kinetic model is confirmed by the small differences between the calculated and the experimental waves. Similar results were obtained when $\tau$ was fixed at $1 \mathrm{~ns}$ or at $1 / k$ in the quenching experiments.

In the experiments with xanthone all the energy absorbed by laser excitation was deposed in $94.4 \mathrm{~ns}$, in just one decay $\phi=1$. The second decay of the deconvolution was found to be redundant. This corresponds to the fast formation of the triplet and its decay with a lifetime in very good agreement with the lifetime obtained by transient absorption. The waves collected in the presence of a quencher could also be described by just one decay, corresponding entirely to the mechanism presented in Scheme $1 \mathrm{~b}$. The rates obtained for the process are shown in Table 2, $\left(\tau_{\mathrm{PAC}}\right)$, as well as the fractions of heat deposed $(\phi$ PAC $)$.

The deconvolution procedure yielded a value of $67.8 \pm$ $0.6 \mathrm{kcal} \mathrm{mol}^{-1}$ for the biphenyl triplet energy and $66.8 \pm 0.5$ $\mathrm{kcal} \mathrm{mol}^{-1}$ for fluorene. The spectroscopic triplet energy of flourene is $67.4 \mathrm{kcal} \mathrm{mol}^{-1}$, which is the same as the triplet energy measured by PAC, within the combined errors of the two measurements. On the other hand, the relaxed triplet energy of biphenyl measured by PAC is $2.3 \mathrm{kcal} \mathrm{mol}^{-1}$ higher than the spectroscopic value. We point out that the triplet energy measured by PAC is that which is directly relevant to bimolecular energy transfer reactions. The triplet energy of biphenyl is less than mid-way between the energies of phosphorescence emission and $\mathrm{T}_{1} \leftarrow \mathrm{S}_{0}$ absorption, meaning that the potential curve for the triplet state is stepper than that of the ground state, as expected from the geometry of those states.

\section{Conclusion}

The triplet energy of fluorene given by PAC (66.8 \pm $0.5 \mathrm{kcal} \mathrm{mol}^{-1}$ ) is in very good agreement with the spectroscopic value. However, the triplet energy of biphenyl measured by PAC $\left(67.8 \pm 0.6 \mathrm{kcal} \mathrm{mol}^{-1}\right)$ is $2.3 \mathrm{kcal} \mathrm{mol}^{-1}$ higher than the spectroscopic value. It seems to be a paradox to measure relaxed triplet energies by PAC and obtain higher energies than using spectroscopic methods. In fact, the phosphorescence also takes place from the relaxed triplet, but leaves the ground state in a nearly planar conformation, $2.3 \mathrm{kcal} \mathrm{mol}^{-1}$ higher in energy than the relaxed ground state. Older literature, in particular the work by Kira and Thomas, had already pointed out the correct triplet energy of biphenyl. Unfortunately their work did not attract the visibility it deserved. We hope that the direct measurement of the biphenyl triplet energy reported in this work will contribute to the use of the correct biphenyl triplet energy, in particular in the polyphenylene polymer field.

\section{Acknowledgements}

We thank Fundação para a Ciência e Tecnologia (FCT, Portugal) and FEDER (European Union) for financial support; project no. POCTI/QUI/47267/2002. C.S. acknowledges FCT for grant SFRH/BPD/13297/2003.

\section{References}

[1] A. Imamura, R. Hoffmann, J. Am. Chem. Soc. 90 (1968) 5379-5385.

[2] F. Negri, M.Z. Zgierski, J. Chem. Phys. 97 (1992) 7124-7136.

[3] I. Hoong-Sun, E.R. Bernstein, J. Chem. Phys. 88 (1988) 7337-7347.

[4] I.B. Berlman, J. Chem. Phys. 52 (1970) 5616-5621.

[5] Y. Gondo, J. Chem. Phys. 41 (1964) 3928-3938.

[6] P.J. Wagner, J. Am. Chem. Soc. 89 (1967) 2820-2825.

[7] A.P. Marchetti, D.R. Kearns, J. Am. Chem. Soc. 89 (1967) 768-777.

[8] P.J. Wagner, Mol. Photochem. 1 (1969) 71.

[9] F. Gessner, J.C. Scaiano, J. Am. Chem. Soc. 107 (1985) 7206-7207.

[10] A. Kira, J.K. Thomas, J. Phys. Chem. 78 (1974) 196-199.

[11] P.E. Poston, J.M. Harris, J. Photochem. Photobiol. A Chem. 60 (1991) $51-61$. 
[12] A.P. Monkman, H.D. Burrows, L.J. Hartwell, L.E. Horsburgh, I. Hamblett, S. Navaratnam, Phys. Rev. Lett. 86 (2001) 1358-1361.

[13] S. Geetha, D.C. Trivedi, Synth. Met. 148 (2005) 187-194.

[14] S. Tepavcevic, A.T. Wroble, M. Bissen, D.J. Wallace, Y. Choi, L. Hanley, J. Phys. Chem. B 109 (2005) 7134-7140.

[15] S.-F. Lim, R.H. Friend, I.D. Rees, J. Li, Y. Ma, K. Robinson, A.B. Holmes, E. Hennebicq, D. Beljonne, F. Cacialli, Adv. Funct. Mater. 15 (2005) 981-988.

[16] K. Brunner, A.v. Dijken, H. Borner, J.J.A.M. Bastiaansen, N.M.M. Kiggen, B.M.W. Langeveld, J. Am. Chem. Soc. 126 (2004) 6035-6042.

[17] S.L. Murov, I. Carmichael, G.L. Hug, Handbook of Photochemistry, second ed., Marcel Dekker, New York, 1993.

[18] R. Dabestani, I.N. Ivanov, Photochem. Photobiol. 70 (1999) 10-34.

[19] S.E. Braslavsky, G.E. Heibel, Chem. Rev. 92 (1992) 1381-1410.

[20] L.G. Arnaut, R.A. Caldwell, J.E. Elbert, L.A. Melton, Rev. Sci. Instrum. 63 (1992) 5381-5389.

[21] G.N. Lewis, M. Kasha, J. Am. Chem. Soc. 66 (1944) 2100-2116.

[22] C. Serpa, L.G. Arnaut, J. Phys. Chem. A 104 (2000) 11075-11086.

[23] J.L. Goodman, K.S. Peters, H. Misawa, R.A. Caldwell, J. Am. Chem. Soc. 108 (1986) 6803-6805.
[24] T. Ni, R.A. Caldwell, L.A. Melton, J. Am. Chem. Soc. 111 (1989) $457-$ 464.

[25] C.M. Brennan, R.A. Caldwell, Photochem. Photobiol. 53 (1991) 165168.

[26] H.D. Burrows, J.S.d. Melo, C. Serpa, L.G. Arnaut, A.P. Monkman, I. Hamblett, S. Navaratatnam, J. Chem. Phys. 115 (2001) 96019606.

[27] H.D. Burrows, J.S.d. Melo, C. Serpa, L.G. Arnaut, M.d.G. Miguel, A.P. Monkman, I. Hamblett, S. Navaratatnam, Chem. Phys. 285 (2002) 3-11.

[28] C. Serpa, P.J.S. Gomes, L.G. Arnaut, S.J. Formosinho, J. Pina, J.S.d. Melo, Chem. Eur. J. 12 (2006) 5014-5023.

[29] N. Chattopadhyay, C. Serpa, L.G. Arnaut, P. Purkayastha, S.J. Formosinho, Phys. Chem. Chem. Phys. 3 (2001) 70-73.

[30] N. Chattopadhyay, C. Serpa, L.G. Arnaut, S.J. Formosinho, Phys. Chem. Chem. Phys. 3 (2001) 3690-3695.

[31] D.E. Damschen, C.D. Merritt, D.L. Perry, G.W. Scott, L.D. Talley, J. Phys. Chem. 82 (1978) 2268-2272.

[32] S.-Y. Hou, W.M. Hetherington III, G.M. Korenowski, K.B. Eisenthal, Chem. Phys. Lett. 68 (1979) 282-284. 